\title{
Study of a transient siphon flow in a cold loop
}

\author{
J. G. Doyle ${ }^{1}$, Y. Taroyan ${ }^{1,2}$, B. Ishak ${ }^{1}$, M. S. Madjarska ${ }^{3, \star}$, and S. J. Bradshaw ${ }^{4}$ \\ 1 Armagh Observatory, College Hill, Armagh BT61 9DG, Northerm Ireland \\ e-mail: jgd@star.arm.ac.uk \\ 2 Solar Physics and Upper-Atmosphere Research Group, Department of Applied Mathematics, University of Sheffield, Sheffield, \\ S3 7RH, UK \\ 3 Solar Influences Data analysis Center, Royal Observatory of Belgium, 3 Circular Avenue, 1180 Brussels, Belgium \\ 4 Space \& Atmospheric Physics, Blackett Laboratory, Imperial College London, Prince Consort Road, London SW7 2BZ, UK \\ Received 10 November 2005 / Accepted 26 February 2006
}

ABSTRACT

\begin{abstract}
Context. The nature of loops is still a matter of debate with several explanations having been put forward. Simultaneous spectral and imaging data have the capacity to provide a new insight into mass motions, dynamics and energetics of loops.

Aims. We report on spectral data taken with the Solar Ultraviolet Measurements of Emitted Radiation spectrograph (SUMER) and imaging data from the Transition Region and Coronal Explorer (TRACE) of a transient event which occurred in a cold loop, lasting a few minutes.

Methods. A sequence of TRACE images in the $1550 \AA$ and $171 \AA$ filters show a disturbance which originated at one foot-point and propagates along the loop. The SUMER slit was placed at the other foot-point of the loop. In order to interpret the results, numerical simulations were performed with the results then converted into observable quantities and compared with the data.

Results. During the event a radiance increase and a relative red shift of $\approx 20 \mathrm{~km} \mathrm{~s}^{-1}$ was detected in the N v $1238.82 \AA$ line. 1D numerical simulations are performed and observable quantities derived from the results of the simulations. The observed dynamic behaviour of the N v $1238.82 \AA$ line profiles was recovered.

Conclusions. The results suggest that the observations could be interpreted in terms of a short-lived siphon flow reaching a speed of $120 \mathrm{~km} \mathrm{~s}^{-1}$ and driven by a nonlinear heating pulse. The energies required to drive the observed red-shifts are estimated to be about $10^{25} \mathrm{erg}$. The absence of a significant blue-shift caused by the return flow is explained.
\end{abstract}

Key words. Sun: corona - Sun: transition region - line: profiles - methods: observational - methods: numerical - hydrodynamics

\section{Introduction}

Highly dynamic loops have been observed at transition region and coronal temperatures since Skylab observations. Mariska \& Dowdy (1992), in their search for Doppler shifts in the Ne VIII $465 \AA$ emission line, reported on down-flow velocities of up to $70 \mathrm{~km} \mathrm{~s}^{-1}$ in active regions while no significant flows were found in a quiet sun region. The Solar Heliospheric Observatory (SoHO) measurements with the Coronal Diagnostic Spectrometer (CDS) in OV $629 \AA$ by Brekke et al. (1997) showed the presence of blue-shifts greater than $50 \mathrm{~km} \mathrm{~s}^{-1}$ in an active region loop with the shift extending over a large fraction of the loop. Winebarger et al. (2001) presented an analysis of continuous intermittent outflow in a bundle of active region coronal loops seen in TRACE data. The measured flow speeds were between 5 and $20 \mathrm{~km} \mathrm{~s}^{-1}$. In a follow-up study, Winebarger et al. (2002) found line-of-sight flows along active region loops of up to $40 \mathrm{~km} \mathrm{~s}^{-1}$ by analyzing co-aligned data from TRACE and SUMER. More recently Teriaca et al. (2004) detected a supersonic siphon-like flow of $130 \mathrm{~km} \mathrm{~s}^{-1}$ in a small quiet sun loop in O VI $1032 \AA$ using SUMER.

The main difficulty in detecting flows in coronal loops with spectrometers (SUMER, CDS) is related to the fact that the Doppler shift can only be measured along the line-of-sight and may cancel out in data with insufficient spatial resolution. On

^ Now at: Max-Planck-Institut fúr Sonnensystemforschung, MaxPlanck-Str. 2, 37191 Katlenburg-Lindau, Germany. the other hand, if high resolution images (e.g. TRACE) are used, then only inhomogeneous flow structures can be detected, whereas loops with steady-state flows appear similar to static loops (Aschwanden 2004). This is why the nature of these loops is still a matter of debate with several explanations having been put forward. Flows can be driven by asymmetries (such as heating or pressure imbalances) between the two legs of the loop (e.g. Boris \& Mariska 1982; Spadaro et al. 1991; Robb \& Cally 1992; Orlando et al. 1995) or by radiatively-cooling condensations (Reale et al. 1996; Müller et al. 2003).

Previous theoretical studies of siphon flows in coronal loops have mainly dealt with steady-state flows. The subject of the present paper is the study of a transient event in a cold loop which lasted for a few minutes. The observations which were carried out with TRACE and SUMER are presented in the first part of the paper. In the second part, a theoretical model for the study of the observed dynamic loop is presented. The evolution of the hydrodynamic quantities inside the loop is examined. Theoretical line profiles are synthesized and the results are compared with the observations. The study allows us to interpret the observations, understand the origin of the transient event and to estimate the energies involved.

\section{Observational data}

\subsection{SUMER}

The SUMER spectrograph (Wilhelm et al. 1995; Lemaire et al. 1997) onboard SoHO was designed to provide line profile 
measurements at a spatial resolution of 1 ". 5 with $0.045 \AA$ spectral pixels as well as a wide spectral coverage ranging from less than 500 to $1610 \AA$.

The data set used here was taken on 1999 June 3 starting at 09:18 UT and ending at 11:08 UT. Slit $0.3^{\prime \prime} \times 120^{\prime \prime}$ was used, exposing for $25 \mathrm{~s}$ on the bottom part of detector B for C I $1249.00 \AA, M g$ x $624.95 \AA$ (in second order) blended with Si II $1250.09 \AA$, O V $629.73 \AA$ (in second order), Si II $1250.41 \AA$ + C I $1250.41 \AA+$ S II $1250.58 \AA$ blend, Si II $1251.16 \AA+$ C I $1251.17 \AA$ blend, N v $1238.82 \AA$ and N V $1242.80 \AA$. The temporal sequence consists of spectral windows of 120 spatial $\times$ 50 spectral pixels. The solar rotation compensation mode was turned on for these observations.

SUMER data reduction involves several steps. We decompressed and reversed the raw data, applying a flat field correction to correct the non-uniformities in the sensitivity of the detector. A geometrical distortion correction was also applied so that the rest position of the line profiles is on the correct spectral pixel and the slit images are straightened. To further analyze the data set, we have used a Gaussian-fitting procedure in the SolarSoft $(\mathrm{SSW})^{1}$ library to get the amplitude, central position, full width at half maximum (FWHM) and $\chi^{2}$ of the line profiles. Due to instrumental broadening, the FWHM needs to be corrected by applying the con_width_funct_3.pro routine. A small drift correction to the Doppler shift over the 110 min observations (due to thermal deformations) was also applied. The derived Doppler shifts are therefore relative to the mean value summed over the full observational interval. As shown by Teriaca et al. (1999), Doyle et al. (2002) and others, the absolute Doppler shift at these temperatures, could be at least $10 \mathrm{~km} \mathrm{~s}^{-1}$ higher than the values given here.

\subsection{TRACE}

TRACE (Handy et al. 1999) is a NASA Small Explorer mission devoted to studying the evolution and propagation of fine-scale magnetic fields and plasma structures throughout the solar atmosphere. The data used here was obtained in the $171 \AA$ and $1550 \AA$ filters starting at 10:03 UT and finishing at 11:00 UT on 1999 June 3, although data was recorded from 07:59 UT. The integration time of each image was $4.1 \mathrm{~s}$ for the $171 \AA$ filter and $5.8 \mathrm{~s}$ for $1550 \AA$, each with a cadence of $60 \mathrm{~s}$ and a pixel size of 0.5 . The instrument was pointed at coordinates Solar_X $=27$ " and Solar_Y $=242^{\prime \prime}$. An overview of the region is given in Fig. 1.

\section{3. $C D S$}

The CDS (Harrison et al. 1995) also onboard SoHO, was designed to obtain spectroheliograms in a number of lines simultaneously in the extreme ultraviolet (EUV) region of the electromagnetic spectrum. CDS has two spectrometers, namely, the Grazing Incidence Spectrometer (GIS) and the Normal Incidence Spectrometer (NIS). Our data set, observed on 1999 June 3 was obtained with NIS.

We use the $244^{\prime \prime} \times 240^{\prime \prime}$ O V $629 \AA$ and Mg IX $368 \AA$ context rasters (taken before the SUMER dataset) using the $4^{\prime \prime} \times 240^{\prime \prime}$ slit. The standard CDS data reduction routines were applied to clean the data of cosmic rays, debias, flat-field and to correct the offset between NIS1 and NIS2.

\footnotetext{
${ }^{1}$ http://www.lmsal.com/solarsoft/
}

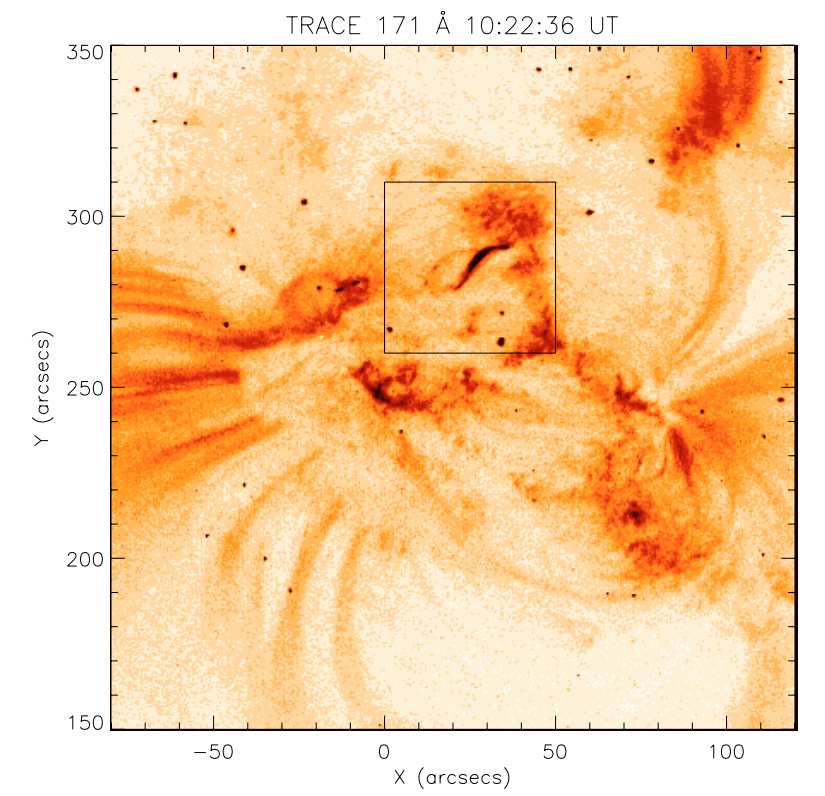

Fig. 1. An overview of the active region as seen in TRACE $171 \AA$ at 10:22 UT on June 3 1999. The small region out-lined by the square box is the subject of the present study.

\subsection{Alignment}

The first step to determining the location of the SUMER slit in the TRACE field of view was to co-register the TRACE $171 \AA$ image taken at 08:25 UT to the Mg IX $368 \AA$ CDS context raster via cross-correlation. Since the CDS raster lasts for about an hour (07:56-08:57 UT), we chose a TRACE image that lies in between this time interval. Secondly, also via cross-correlation, we found the match of the SUMER O v $629 \AA$ radiance profile along the slit (in both original and degraded to the CDS spatial resolution) in one of the South-North cross-sections of the O v $629 \AA$ CDS context raster. Next, by putting the slit on CDS Mg IX $368 \AA$ A context raster, we deduced its location on the TRACE image.

With this co-alignment of CDS-TRACE plus CDS-SUMER, we get the pointing of SUMER over TRACE, although not $100 \%$ accurate. The uncertainty is at least $4^{\prime \prime}$ i.e. the width of the CDS slit. Moving the SUMER slit around on the TRACE image by certain offsets as well as visually inspecting the features on TRACE, we manage to identify its location on TRACE as shown in Fig. 2.

\section{Results}

The present paper is concerned with a transient event which occurred in a particular loop. The foot-point coordinates of the loop are Solar_X $=20^{\prime \prime}$, Solar_Y $=280^{\prime \prime}$ and Solar_X $=36^{\prime \prime}$, Solar_Y $=292^{\prime \prime}$. The transient brightening of the loop which took place between 10:21 UT and 10:25 UT can be seen in TRACE $1550 \AA$ and TRACE $171 \AA$ (Fig. 2). Both filters show that the brightening initially appears at the left foot-point, moves towards the right foot-point and eventually fades away. At the same time, the SUMER data show a radiance increase in $\mathrm{N} \mathrm{V}$ $1238 \AA$ and a red-shift at the right foot-point where the slit is located. In Fig. 3 we show N v SUMER images at two times, 10:20:16 UT (i.e. before the event started) and 10:21:12 UT (i.e. around time of maximum line shift and brightness). These can be best seen in the second and third columns which 

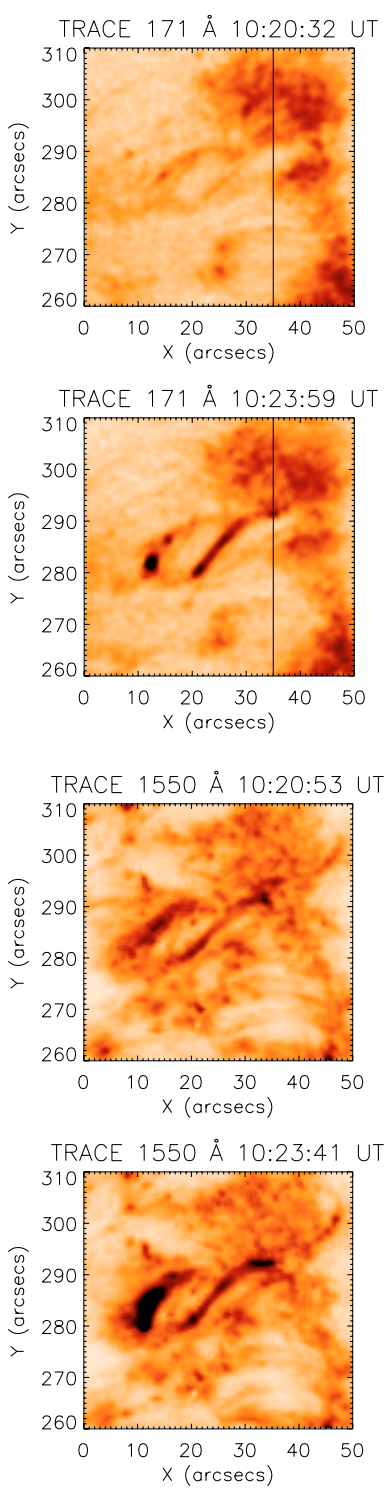
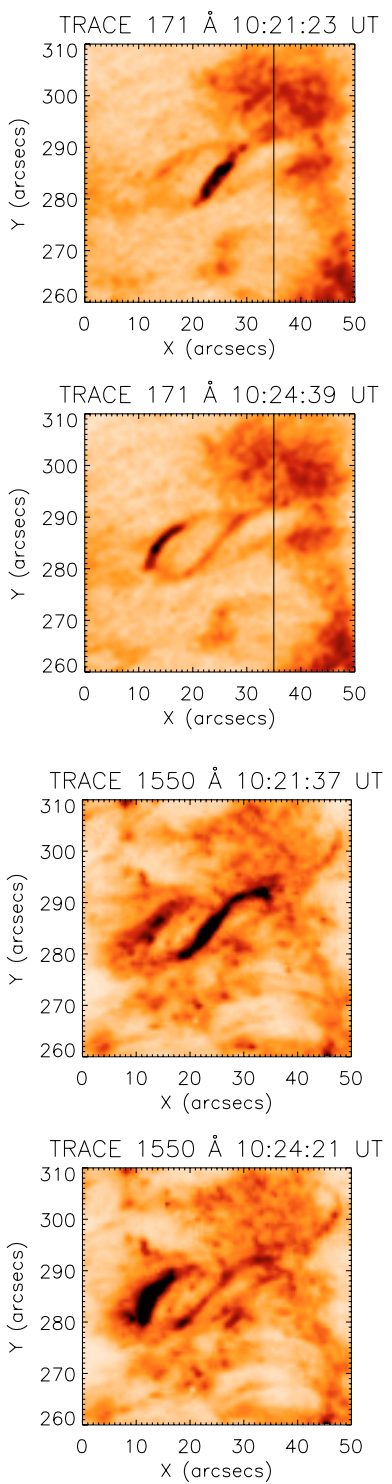
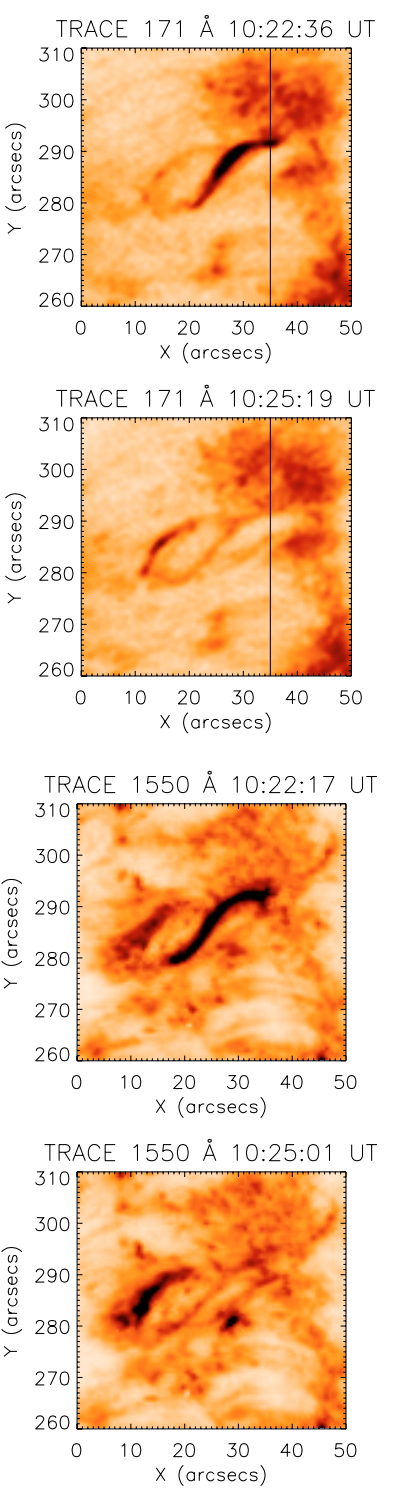
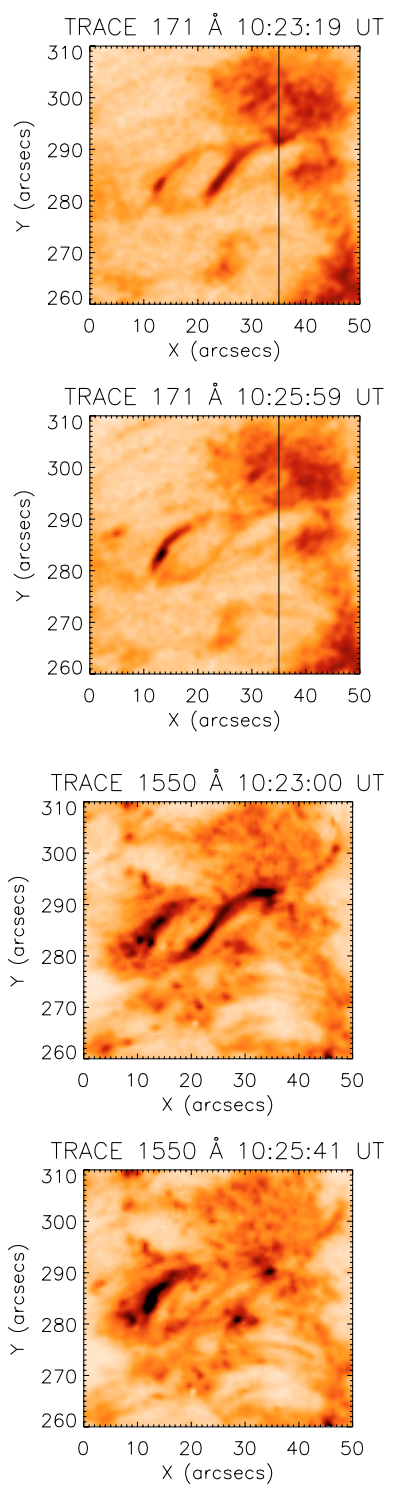

Fig. 2. A sequence of TRACE images in the $1550 \AA$ and $171 \AA$ filters. The position of the SUMER slit is outlined on the $171 \AA$ image.

correspond to $Y=287^{\prime \prime}$ and $Y=289^{\prime \prime}$, although the event is presence stretching from $Y=285^{\prime \prime}$ to $291^{\prime \prime}$. In Fig. 4 we show the time-line of the radiance increase and the Doppler shift. The radiance increase at maximum is $\approx 15-20$ times the quiescent value taken a few minutes before the event at the same location. It then subsequently decreases as the loop returns to its initial quiet state. The data taken at $Y=287^{\prime \prime}$ shows a relative red-shift of $\approx 20 \mathrm{~km} \mathrm{~s}^{-1}$ which lasts for just over a minute. In the case of the data at $Y=289^{\prime \prime}$ a similar red-shift is followed by a smaller blue-shift. The subsequent evolution of the loop as seen by SUMER is characterized with low intensities and small Doppler shifts. In TRACE, the evolution is better seen in the $1550 \AA$ filter where the length of the loop is well defined as shown at times 10:21:37-10:23:41 UT in Fig. 2. The loop subsequently disappears but the intense brightening at the right foot-point seen at time 10:25:41 UT persists for $80 \mathrm{~s}$ longer. The plasma is estimated to move along the loop at a velocity of at least $100 \mathrm{~km} \mathrm{~s}^{-1}$.

The $\mathrm{Mg} \mathrm{X} / \mathrm{Si}$ II blend also shows a response to the brightening, perhaps by a factor of three as shown by Fig. 5 . The cold lines like C I do not show the event, with $\mathrm{Si} \mathrm{X}$ in second order responding a bit. This therefore suggests that the event just reached temperatures of $\approx 1 \mathrm{MK}$. This is consistent with the event being observable in the EIT $195 \AA$ image, which at these temperatures is mostly due to Fe XII, although for temperatures less than $1 \mathrm{MK}, \mathrm{Fe}$ VIII can be dominant. The feature at $1251.70 \AA$ shows a large response to the event, suggesting that it is a hot line. Possible identifications include Si V $1251.39 \AA$ and S IV $1251.64 \AA$, however, both of these are extremely weak lines (verified using CHIANTI). Therefore we suggest that it is O IV $625.853 \AA$ in second order. This would therefore imply the presence of another second order O IV line at $1250.25 \AA$, close to the $\mathrm{Mg} \mathrm{X}$ second order line. Using CHIANTI with a quiet sun DEM gives a radiance of $1.1 \mathrm{erg} \mathrm{cm}^{-2} \mathrm{~s}^{-1} \mathrm{sr}^{-1}$ for O IV $625.853 \AA$ compared to $14.5 \mathrm{erg} \mathrm{cm}^{-2} \mathrm{~s}^{-1} \mathrm{sr}^{-1}$ for Mg X $624.94 \AA$. Taking the N v enhancement factor of 20 , the factor of 3 enhancement for $\mathrm{Mg}$ X suggests that the lines should be in the ratio of 2:1 in favour of $\mathrm{Mg} \mathrm{X}$, which is in agreement with Fig. 5.

In the next section, a 1D hydrodynamic loop model is introduced. Numerical simulations are performed with the results then converted into observable quantities and compared with the data in order to interpret the observations. 

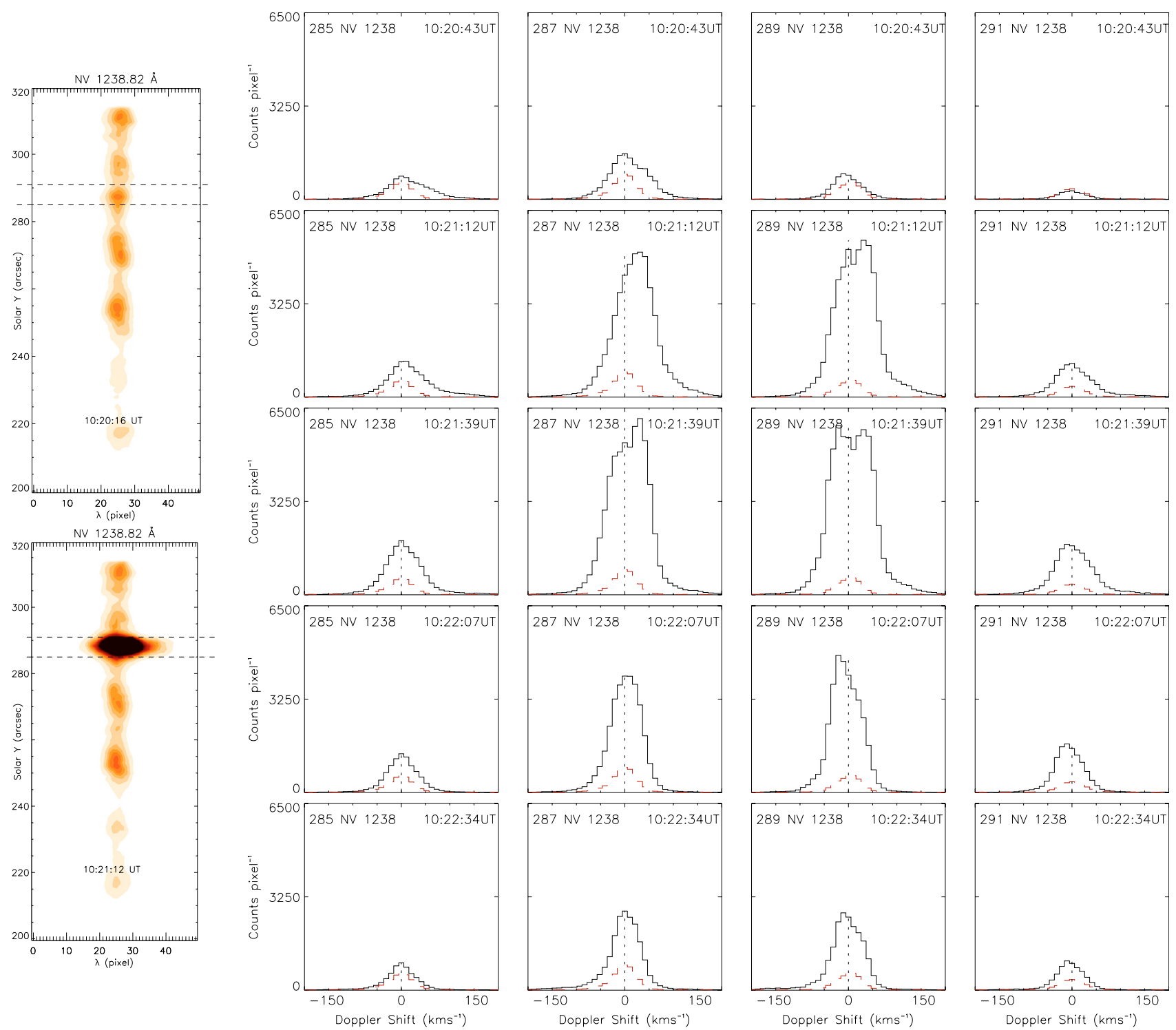

Fig. 3. The left two panels shows SUMER spectra in N v $1238 \AA$ at two times 10:20:16 and 10:21:12 UT. The event occurred between $Y=285^{\prime \prime}$ and $Y=291^{\prime \prime}$. The wavelength scale is in pixels where 1 pixel is $0.0436 \AA$. The panels on the right shows the time sequence of $\mathrm{N} v$ spectra from $Y=285^{\prime \prime}$ to $291^{\prime \prime}$ during the rise and early decay phase of the loop (solid line) and a quiet Sun profile (dashed line) taking at the same time but shifted a few pixels from the brightening.

\section{Numerical modelling and interpretation of the observed loop dynamics}

\subsection{Initial setup}

The length of the loop as inferred from the TRACE images is about $20 \mathrm{Mm}$. The exact temperature profile of the loop is not known. However, the fact that during the transient flow the loop is initially clearly visible in TRACE $1550 \AA$ means that it has a temperature of around $100000 \mathrm{~K}$. We consider a $20 \mathrm{Mm}$ long gravitationally stratified 1D loop with foot-points anchored in the deep chromosphere. The loop is a semi-circle with a vertical loop plane, i.e., the inclination angle is zero. For simplicity, a uniform heating with a heating rate of $\mathcal{H}=$ $1.5 \times 10^{-5} \mathrm{erg} \mathrm{cm}^{-3} \mathrm{~s}^{-1}$ is applied along the loop to balance the losses due to thermal conduction and optically thin radiation. The loop is initially in hydrostatic equilibrium and has a maximum temperature of $T=250000 \mathrm{~K}$ at the apex. The conventional way of calculating optically-thin radiative energy loss is to use power-law fits to a radiative loss curve over a range of temperatures. It would be unphysical to extend this loss curve below $20000 \mathrm{~K}$ because at these temperatures the chromosphere is optically-thick to many wavelengths. Therefore, for the boundary conditions we choose a footpoint temperature of $20000 \mathrm{~K}$. This represents a physically reasonable lower boundary condition in the absence of a detailed knowledge and thorough treatment of the deep chromosphere and photosphere. The equilibrium is derived by integrating the $1 \mathrm{D}$ hydrostatic equations of pressure and energy. The only coordinate is the distance along the loop $s$. The left foot-point is placed at the origin $s=0$, i.e, $0<s<L$, where $L=20 \mathrm{Mm}$.

\subsection{Hydrodynamic evolution of the loop}

The disturbance seen in the series of TRACE images (Fig. 2) originates at the left foot-point of the loop and travels towards 

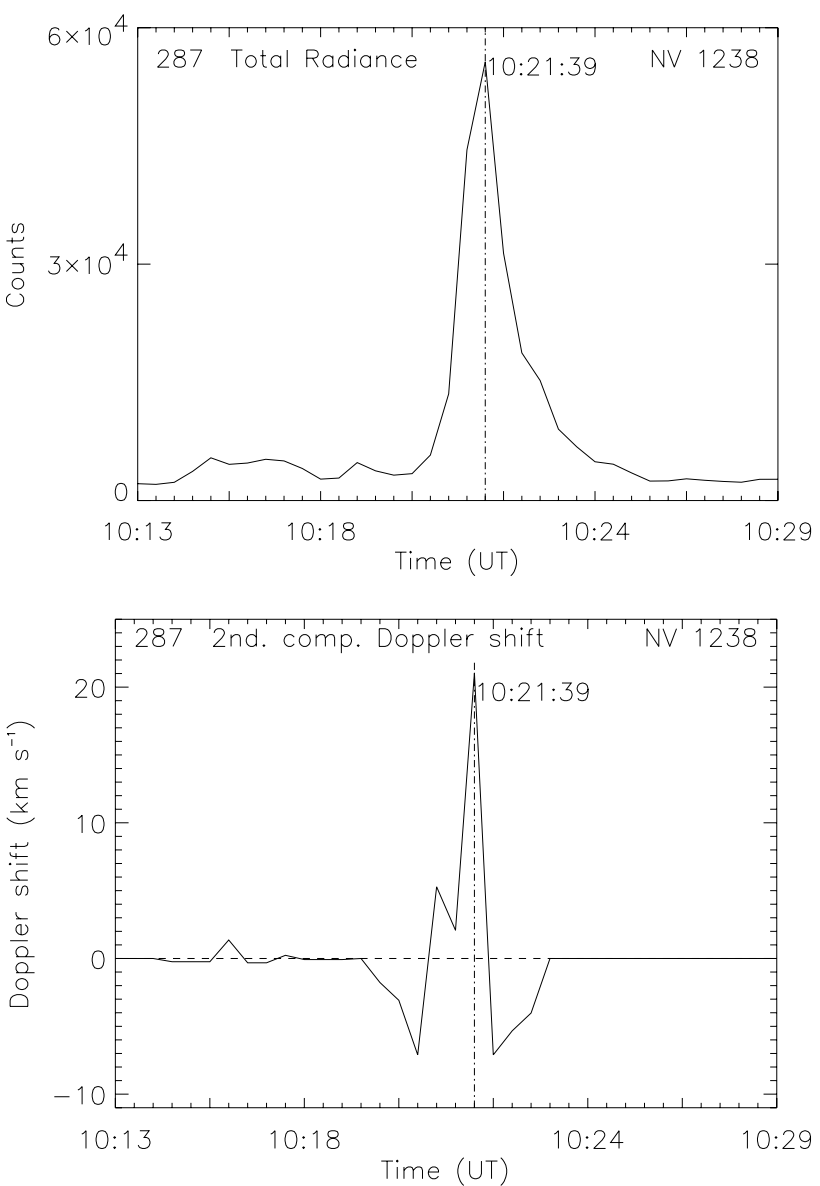

Fig. 4. The total radiance and Doppler shift at pixel $287^{\prime \prime}$.

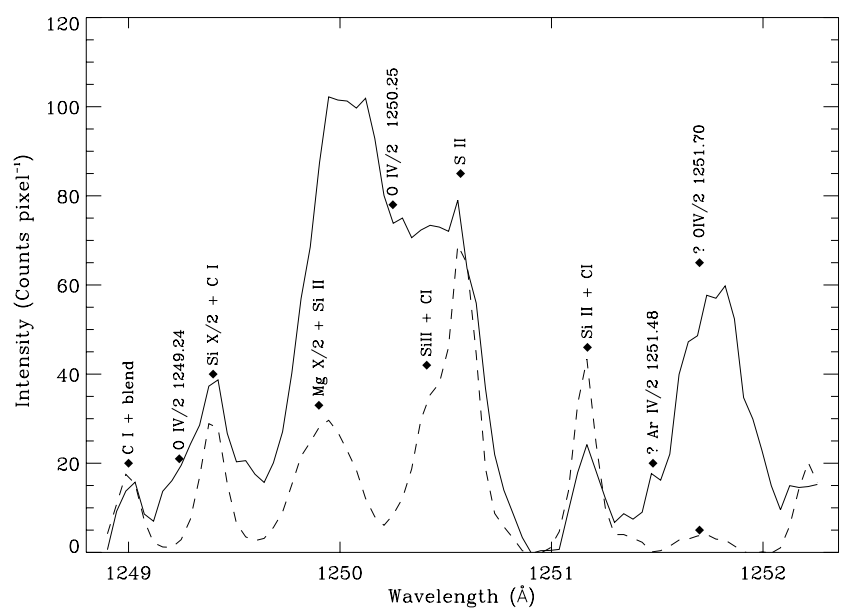

Fig. 5. The section of SUMER spectrum showing the enhancements in second order Mg X $624.9426 \AA$, and the first order lines of C I, S II and Si II. Note the unknown feature at $1251.76 \AA$ which we believe is due to second order O IV $625.853 \AA$ (see text).

the right foot-point. This disturbance is modelled as a heating pulse which has the following form:

$h= \begin{cases}h_{0} \sin \left(\frac{\pi t}{P}\right) \exp \left(-\frac{s-s_{0}}{s_{h}}\right), & 0 \leq t \leq P \\ 0, & t>P,\end{cases}$

where $h_{0}=0.03 \mathrm{erg} \mathrm{cm}^{-3} \mathrm{~s}^{-1}$ is the maximum heat input, $s_{0}$ and $s_{h}$ are the location of maximum heating and the heating scale
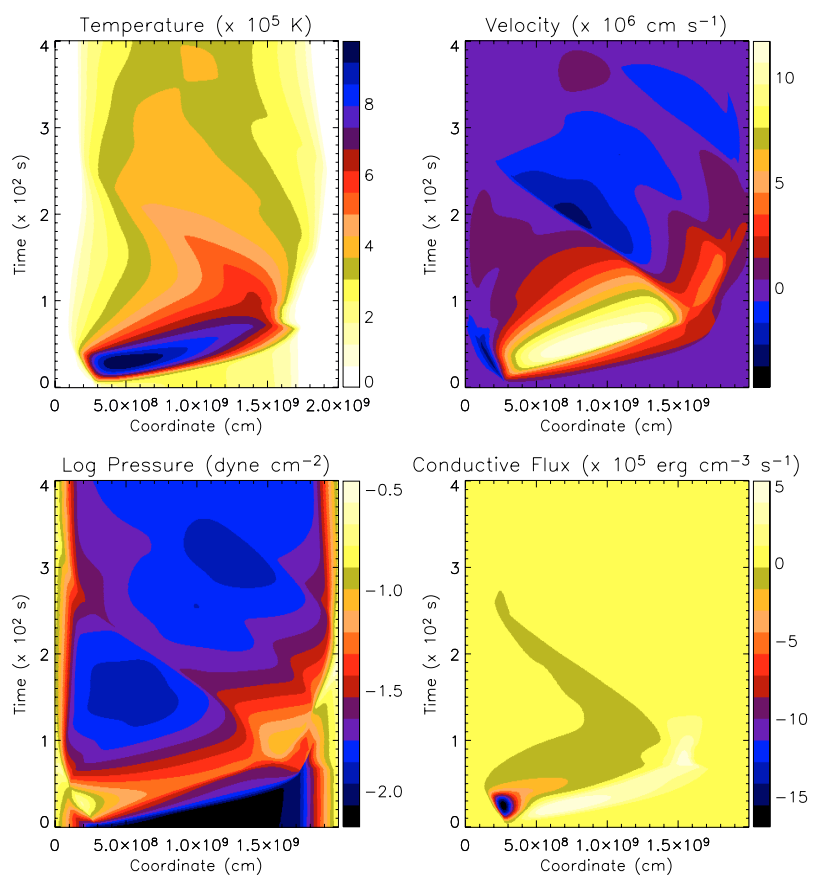

Fig. 6. The evolution of temperature, velocity, pressure and conductive flux inside the loop from $t=0 \mathrm{~s}$ to $t=400 \mathrm{~s}$. Dark colours indicate high temperature, negative velocities, low pressure and negative flux, whereas bright colours indicate low temperature, positive velocities, high pressure and positive flux.

length. The maximum heat input is fixed in the lower transition region near the left foot-point at $s_{0}=3 \times 10^{8} \mathrm{~cm}(3 \mathrm{Mm})$. The heating scale length is $s_{h}=10^{8} \mathrm{~cm}(1 \mathrm{Mm})$. The duration of the heat input is $P=40 \mathrm{~s}$. The heating pulse in Eq. (1) is added to the background uniform heating $\mathcal{H}$. The heating terms are included in the $1 \mathrm{D}$ hydrodynamic equation of energy. The heating pulse may lead to high flow speeds and the ion populations may significantly depart from equilibrium. Therefore, the effects of a nonequilibrium ionisation balance are taken into account by coupling the hydrodynamic equations governing the conservation of mass, momentum and energy with the time-dependent ionisation balance equations. We use the numerical code HYDRAD (Bradshaw \& Mason 2003; Bradshaw et al. 2004) to solve this system of differential equations which are coupled via the feedback of the time-dependent ion populations into the radiative loss term in the energy equation. HYDRAD has an adaptive computational grid which allows us to accurately trace the position of the transition region as the loop evolves.

The time-distance plots in Fig. 6 display the hydrodynamic evolution of the loop. The heating pulse results in a temperature increase near the left foot-point. By $t=28 \mathrm{~s}$ the temperature reaches a maximum value of about $1 \mathrm{MK}$. Some part of the heating pulse is converted into kinetic energy. The resulting plasma flow reaches a maximum speed of about $120 \mathrm{~km} \mathrm{~s}^{-1}$ by $t=50 \mathrm{~s}$. The injected energy is carried towards the right foot-point both in the form of kinetic energy and in the form of heating by thermal conduction. Figure 6 shows that the conduction peak moves ahead of the velocity peak. This is due to the large temperature gradient caused by the initial localized heating pulse. The velocity profile gradually steepens as its peak moves faster than the trough due to the higher temperature. As a result, the kinetic energy carried by the plasma flow is very efficiently converted into heating by thermal conduction, the rest being reflected back from the dense chromospheric foot-point of the loop. In this way 
a propagating wave is set up inside the loop. The wave quickly decays due to the action of thermal conduction and radiation and the loop returns to the initial equilibrium. It is possible to select a different set of heating parameters. For example, when the duration of the pulse is increased the loop reaches temperatures in excess of $1 \mathrm{MK}$. On the other hand, if the duration of the heating pulse is increased and the maximum heat input $h_{0}$ is decreased the flow speeds near the right foot-point become small.

\subsection{Comparison of synthesized line profiles with the observations}

The next step in our study is the comparison of the simulation results with the observations: theoretical line profiles are synthesized and compared with the SUMER line profiles. We follow a well-known procedure for the derivation of the line profiles (see, e.g., Spadaro et al. 1990; Sarro et al. 1999). The fractional ion populations $\left(N_{\text {ion }} / N_{\text {elem }}\right)$ as a function of time are calculated by HYDRAD using the ionisation and recombination rates given by Mazzotta et al. (1998). We adopt the set of coronal element abundances given by Feldman (1992) and use the CHIANTI atomic database (Young et al. 2003) to obtain the emissivities for each line of interest. The emissivity of a resonant line per unit interval of wavelength is given by the expression

$E_{\lambda} \propto \frac{h c}{\lambda} \frac{\Omega}{\omega} \frac{N_{1}}{N_{\text {ion }}} \frac{N_{\text {ion }}}{N_{\text {elem }}} \frac{N_{\text {elem }}}{N_{\mathrm{H}}} N_{\mathrm{H}} N_{\mathrm{e}} \frac{\exp \frac{-W}{k_{\mathrm{B}} T}}{\sqrt{T}} \Phi(\lambda)$,

where $h$ is the Planck constant, $c$ is the speed of light, $\Omega$ is the collisional strength, $\omega$ is the statistical weight of the lower level, $N_{1} / N_{\text {ion }}$ is the ratio of ions responsible for the emission in the ground state relative to the total number of ions per unit volume, $N_{\text {ion }} / N_{\text {elem }}$ is the relative abundance of the ionic species, $N_{\text {elem }} / N_{\mathrm{H}}$ is the abundance of the element relative to hydrogen, $N_{\mathrm{H}}$ is the proton density, $N_{\mathrm{e}}$ is the electron density, $W$ is the energy difference between the upper and lower levels, $k_{\mathrm{B}}$ is the Boltzman constant, and

$\Phi(\lambda)=\frac{1}{\Delta \lambda_{0} \sqrt{\pi}} \exp -\left[\frac{\lambda-\lambda_{0}-\lambda_{\mathrm{s}}}{\Delta \lambda_{0}}\right]^{2}$

is the line broadening function. In the above definition $\lambda_{0}$ is the rest wavelength of the resonance line, $\lambda_{\mathrm{s}}=\left(\lambda_{0} / c\right) v_{\mathrm{p}}$ is the Doppler shift corresponding to the bulk velocity of the plasma projected on the line of sight $v_{\mathrm{p}}, \Delta \lambda_{0}$ is the Doppler width of the line:

$\Delta \lambda_{0}=\frac{\lambda_{0}}{c} \sqrt{\frac{2 k_{\mathrm{B}} T}{m_{\mathrm{i}}}+\xi^{2}}$,

$\xi$ is the characteristic non-thermal velocity (assumed to be $25 \mathrm{~km} \mathrm{~s}^{-1}$ ) and $m_{\mathrm{i}}$ the mass of the ion producing the spectral line. In order to calculate the line of sight velocity we have to take into account the heliographic position of the loop as well as the azimuthal angle of the loop foot-point baseline with the north-south direction. These parameters are evaluated using the TRACE images in Fig. 2. Therefore, the following results are only valid for the observed loop. The total radiance of a spectral line integrated along a single pixel is

$I(\lambda, t)=\int_{s_{1}}^{s_{\mathrm{r}}} E(\lambda, t, s) \mathrm{d} s$,

where $s_{1}$ and $s_{\mathrm{r}}$ need to be specified. Figure 2 shows that the SUMER slit is placed near the right foot-point of the loop. We

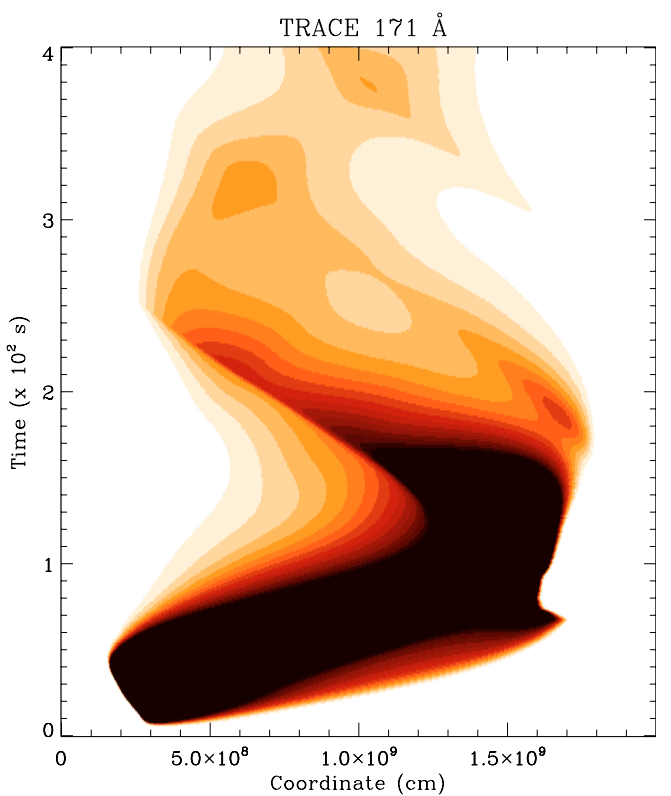

Fig. 7. Synthesized emission in the TRACE $171 \AA$ filter in 1D during the first $400 \mathrm{~s}$ of evolution. Dark regions represent strong emission and white regions correspond to no emission.

set $s_{\mathrm{r}}=18.6 \mathrm{Mm}$. Then $s_{1}$ is determined by the requirement that the projection of the loop segment $\left[s_{1}, s_{\mathrm{r}}\right]$ on the plane normal to the line of sight has a pixel size of $1^{\prime \prime}$ which corresponds to $s_{1}=16.7 \mathrm{Mm}$.

The above described procedure for calculating the line profiles is applied to the resonant N V $1238 \AA$ and O VI $1032 \AA$ lines, plus the TRACE $171 \AA$ filter. In Fig. 7 we plot the synthesized emission in the TRACE $171 \AA$ filter in 1D during the first $400 \mathrm{~s}$ of evolution. The dark regions represent strong emission and the bright regions correspond to weak emission. Figure 7 shows that the brightening of the loop (darker regions) lasts for about three minutes which is consistent with the actual images in the TRACE $171 \AA$ filter shown in Fig. 2.

The synthesized line profiles and the corresponding line shifts for N V $1238 \AA$ and O VI $1032 \AA$ are displayed in Fig. 8 . The radiance is normalized with respect to the radiance of the same line at $t=0 \mathrm{~s}$. The Doppler shift is measured in velocity units $\left(\mathrm{km} \mathrm{s}^{-1}\right)$. The results of the numerical simulations for N v $1238 \AA$ can be compared with the SUMER observations for the same line. The radiance shows no variations and there is no Doppler shift during the first $50 \mathrm{~s}$ after the pulse. As the pulse reaches the slit the radiance increases by about 10 times when $t=68 \mathrm{~s}$, which is slightly less than the SUMER observations. Figure 8 also shows an increase in the line width due to increasing temperature. The radiance increase in the O VI $1032 \AA$ line is more pronounced and the peak occurs at $t=160 \mathrm{~s}$. Thus the peak for N V $1238 \AA$ is followed by the peak for O VI $1032 \AA$ which can be explained by the heating of the plasma. The red-shift corresponding to N V $1238 \AA$ reaches a maximum of $38 \mathrm{~km} \mathrm{~s}^{-1}$, consistent with the SUMER observations. The peak occurs at $t=105 \mathrm{~s}$. Note the presence of smaller local peaks at about $t=60 \mathrm{~s}$ (bottom panels of Fig. 8). This is consistent with the results of the hydrodynamic simulations: it has already been shown that the conduction peak moves ahead of the velocity peak. The cadence of the present dataset is not sufficient to resolve the double flow structure. The first peak corresponds to the arrival of the conduction front, whereas the second higher peak signals 

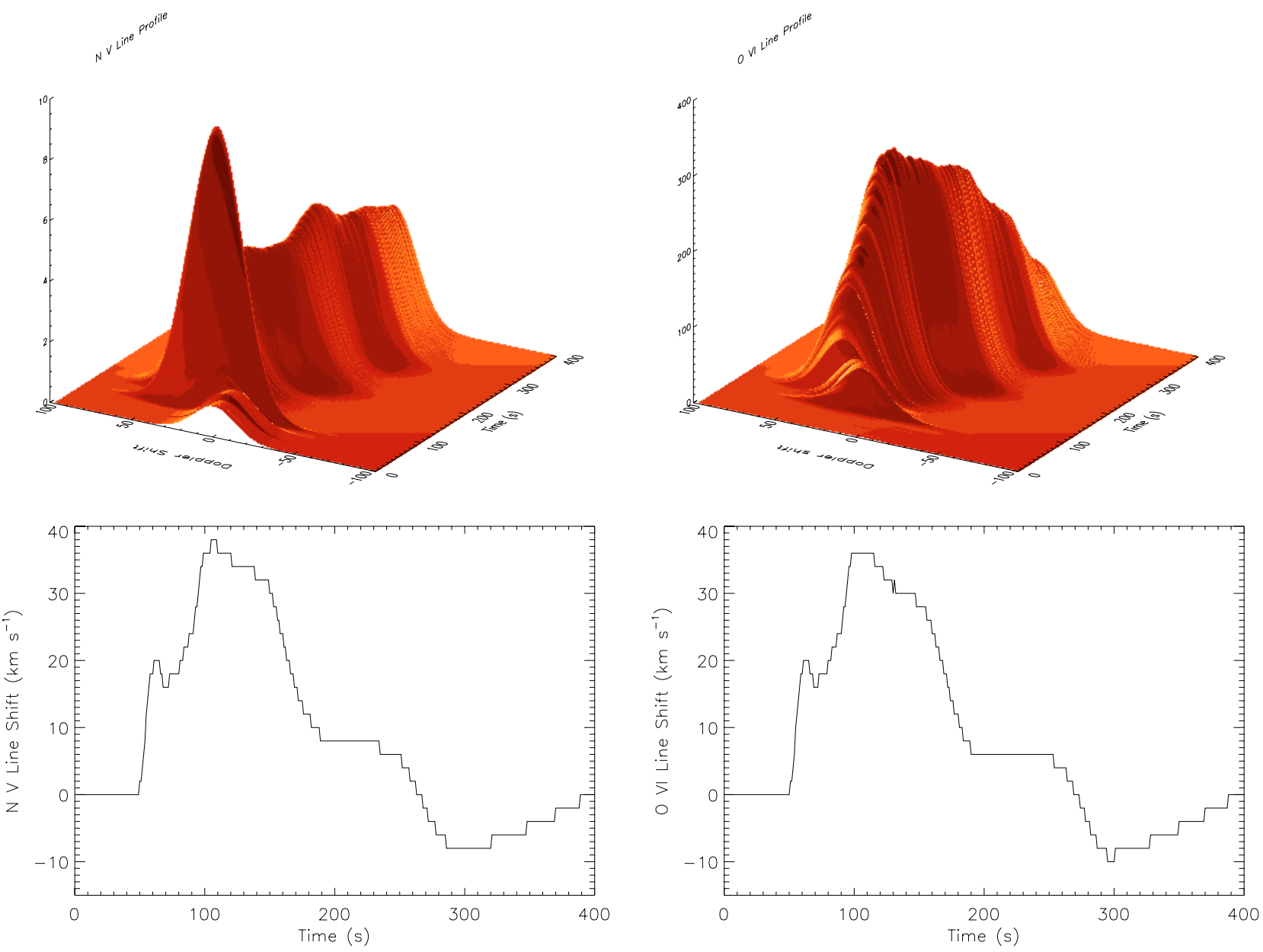

Fig. 8. The line profiles (top) and the corresponding line shifts (bottom) for N v $1238 \AA$ and O VI $1032 \AA$ measured in velocity units $\left(\mathrm{km} \mathrm{s}^{-1}\right)$ during the first $400 \mathrm{~s}$ after the heating pulse. The radiance $I$ is normalized with respect to the radiance at $t=0 \mathrm{~s}$. Positive velocities represent red-shifts and negative velocities represent blue-shifts.

the arrival of the peak of the plasma flow. The large red-shifts $\left(>30 \mathrm{~km} \mathrm{~s}^{-1}\right)$ last just over a minute. These positive red-shifts are then followed by small blue-shifts as the flow is reflected from the dense chromospheric foot-point of the loop. The smallness of the blue-shift could be explained by the fact that the kinetic energy is very efficiently converted into heating due to the steepening of the velocity and the corresponding large conductive flux. The initial line profile is gradually restored.

The similarity of the numerical results with the SUMER (and TRACE) data allows us to interpret the observations as a transient siphon flow in a cold loop driven by a heating pulse at one foot-point of the loop. Based on the results of the numerical study and assuming a loop thickness of $2 \mathrm{Mm}$, we estimate that the amount of energy required to drive the flow is about $10^{25} \mathrm{erg}$.

\section{Conclusions}

We have presented joint TRACE and SUMER observations of a transient event in a cold loop. The data set was taken on 1999 June 3 starting at 09:18 UT and ending at 11:08 UT. The SUMER slit was placed near the foot-point of the loop. A sequence of TRACE images in the $1550 \AA$ and $171 \AA$ filters show that the event started at the opposite foot-point of the loop. It lasted for a few minutes during which a temporary increase in the radiance of $\mathrm{NV} 1238 \AA$ and the corresponding red-shifts reaching $\approx 30 \mathrm{~km} \mathrm{~s}^{-1}$ (i.e. after adding a correction value to the derived relative velocity) were registered. A complementary numerical analysis is carried out in order to interpret the observed dynamic behaviour of the loop and to estimate the energies involved in the transient event. The results show that the observations could be interpreted in terms of a transient siphon flow which may arise due to a heating pulse at the loop foot-point. The numerical analysis also shows that the absence of a significant blue-shift in the SUMER data could be explained by the steepening of the pulse as it travels along the loop from one footpoint to the other. Furthermore, since the maximum temperature reaches $\approx 1 \mathrm{MK}$, this is consistent with the loop being observable in TRACE $171 \AA$ and SUMER Mg X data. The energy required to drive the flow is estimated to be $10^{25} \mathrm{erg}$.

Acknowledgements. We would like to thank the SUMER team at Max Planck Institute for Solar System Research (MPS), plus the CDS and TRACE teams at Goddard Space Flight Center for their help in obtaining the present data. SUMER is part of SoHO, a mission of international cooperation between ESA and NASA. Research at Armagh Observatory is grant-aided by the N. Ireland Dept. of Culture, Arts and Leisure. This work was partially supported by the Program for Research in Irish Third Level Institutions for Grid-enabled Computational Physics of Natural Phenomena (Cosmogrid) and by PPARC grant PPA/G/S/2002/00020. M.M. acknowledge the support of the Belgian Federal Science Policy Office. B.I. wishes to thank E. O'Shea and I. Ugarte-Urra for productive discussions. CHIANTI is a collaborative project involving the NRL (USA), RAL (UK), MSSL (UK), the Universities of Florence (Italy) and Cambridge (UK), and George Mason University (USA). SJB is supported by a 
PPARC Post-Doctoral Fellowship. We thank the referee V. Hansteen for helpful comments.

\section{References}

Aschwanden, M. J. 2004, Physics of the Solar Corona: An Introduction (Berlin: Springer)

Bradshaw, S. J., \& Mason, H. E. 2003, A\&A, 407, 1127

Bradshaw, S. J., Del Zanna, G., \& Mason, H. E. 2004, A\&A, 425, 287

Brekke, P., Kjeldseth-Moe, O., \& Harrison, R. A. 1997, Sol. Phys., 175, 511

Boris, J. P., \& Mariska, J. T. 1982, ApJ, 258, L49

Doyle, J. G., Madjarska, M. S., Roussev, I., Teriaca, L., \& Giannikakis, J. 2002, A\&A, 396, 255

Feldman, U. 1992, Phys. Scripta, 46, 202

Handy, B. N., Acton, L. W., Kankelborg, C. C., et al. 1999, Sol. Phys., 187, 229

Harrison, R. A., Sawyer, E. C., Carter, M. K., et al. 1995, Sol. Phys., 162, 233

Lemaire, P., Wilhelm, K., Curdt, W., et al. 1997, Sol. Phys., 170, 105
Mariska, J. T., \& Dowdy, J. F. 1992, ApJ, 401, 754

Mazzotta, P., Mazzitelli, G., Colafrancesco, S., \& Vittorio, N. 1998, A\&AS, 133, 403

Müller, D. A. N., Hansteen, V. H., \& Peter, H. 2003, A\&A, 411, 605

Orlando, S., Peres, G., \& Serio, S. 1995a, A\&A, 294, 861

Robb, T. D., \& Cally, P. S. 1992, ApJ, 397, 329

Reale, F., Peres, G., \& Serio, S. 1996, A\&A, 316, 215

Sarro, L. M., Erdélyi, R., Doyle, J. G., \& Pérez, M. E. 1999, A\&A, 351, 721

Spadaro, D., Noci, G., Zappalà, R., \& Antiochos, S. K. 1990, ApJ, 355, 342

Spadaro, D., Antiochos, S. K., Spiro, K., \& Mariska, J. T. 1991, ApJ, 382, 338

Teriaca, L., Banerjee, D., \& Doyle, J. G. 1999, A\&A, 349, 636

Teriaca, L., Banerjee, D., Falchi, A., Doyle, J. G., \& Madjarska, M. S. 2004, A\&A, 427, 1065

Wilhelm, K., Curdt, W., Marsch, E., et al. 1995, Sol. Phys., 162, 189

Winebarger, A. R., DeLuca, E. E., \& Golub, L. 2001, ApJ, 553, L81

Winebarger, A. R., Warren, H., van Ballegooijen, A., DeLuca, E. E., \& Golub, L. 2002, ApJ, 567, L89

Young, P. R., Del-Zanna, G., Landi, E., et al. 2003, A\&AS, 144, 135 